\title{
Event horizon scale emission models for Sagittarius A*
}

\author{
J. Dexter \\ Departments of Physics and Astronomy, University of California, Berkeley, CA, USA \\ email: jdexter@berkeley.edu
}

\begin{abstract}
Very long baseline interferometry observations at millimeter wavelengths have detected source structure in Sgr A* on event horizon scales. Near-infrared interferometry will achieve similar resolution in the next few years. These experiments provide an unprecedented opportunity to explore strong gravity around black holes, but interpreting the data requires physical modeling. I discuss the calculation of images, spectra, and light curves from relativistic MHD simulations of black hole accretion. The models provide an excellent description of current observations, and predict that we may be on the verge of detecting a black hole shadow, which would constitute the first direct evidence for the existence of black holes.
\end{abstract}

Keywords. accretion, accretion disks - black hole physics - relativity

\section{Introduction}

The Galactic center black hole, Sagittarius A* (Sgr A*), is the largest in angular size (along with M87), with an event horizon spanning tens of microarcseconds. Recently, 1.3 mm wavelength VLBI observations (the "Event Horizon Telescope" experiment, EHT), have detected source structure in $\mathrm{Sgr} \mathrm{A}^{*}$ with a size of $\simeq 4$ Schwarzschild radii (Doeleman et al. 2008). Similar resolution will be achieved by near-infrared interferometry in the next few years (the VLTI GRAVITY experiment, Eisenhauer et al. 2008). These observations provide a remarkable opportunity to explore strong gravity around black holes. The predicted "shadow" (Bardeen 1973; Falcke et al. 2000) may be detected by future EHT observations. Time-resolved astrometry with VLTI GRAVITY data could map out particle orbits near the event horizon (Hamaus et al. 2009).

Models are required to interpret data from both experiments. These include geometric (Gaussian and annulus surface brightness profiles) and semi-analytic accretion flow models (Broderick et al. 2009, 2011). General relativistic MHD (GRMHD) simulations of black hole accretion (e.g., Gammie et al. 2003; De Villiers \& Hawley 2003) provide a complementary description of the innermost part of the accretion flow. Simulations neglect the photons that astronomers observe, but at the low luminosity of Sgr A* they can accurately (Dibi et al. 2012; Drappeau et al. 2013) be added after the fact by performing radiative transfer on the simulation data. Recently, many authors have used these models to study Sgr A* and M87 (e.g., Noble et al. 2007; Mościbrodzka et al. 2009; Shcherbakov et al. 2012). Here I briefly review the ray tracing radiative transfer method I have used to calculate event horizon scale emission models of $\mathrm{Sgr} \mathrm{A}^{*}$, and the results of comparing these models to observations. I then highlight some important open questions and possible ways to answer them with future EHT and GRAVITY data. 


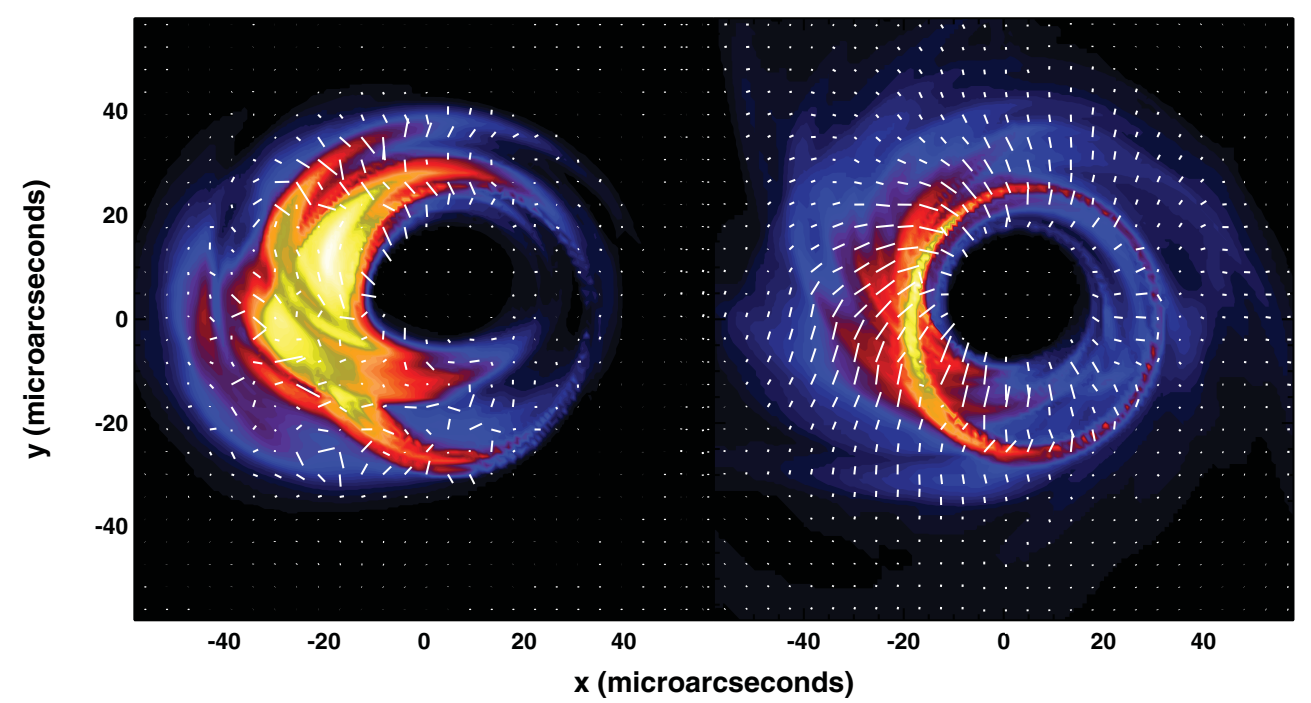

Figure 1. False color accretion disk images and polarization maps from solving the polarized, time-dependent general relativistic radiative transfer equations with input fluid data from state of the art GRMHD simulations (Dexter \& McKinney 2013, in prep.). Depending on the strength of Faraday rotation and conversion effects, the emission can be de-polarized on small scales (left) or remain coherent (right). EHT polarization will therefore be a sensitive probe of the plasma conditions. The left/right image asymmetries are caused by Doppler beaming of light from relativistic orbital motion, and the black hole shadow is clearly visible in the center of both images. These relativistic effects lead to a characteristic crescent shape. [A COLOR VERSion IS AVALLAbLE ONLINE.]

\section{Methods}

The appearance of gas in the immediate vicinity of a black hole is shaped by relativistic effects (see Figure 1), which can be captured using numerical ray tracing radiative transfer calculations (for details see Dexter et al. 2010). Rays are traced from each pixel of a distant observer's camera to the black hole using our analytic code GEOKERR (Dexter \& Agol 2009) for photon trajectories around spinning black holes. The radiative transfer equation is solved along each ray using synchrotron emission and absorption coefficients calculated from simulation data. The basis vectors describing the polarization are parallel transported along each ray analytically using the Penrose-Walker constant (Dexter 2011) and the angles between these vectors and the magnetic field are found by transforming to the orthonormal comoving frame (Shcherbakov \& Huang 2011). This approach is impractical for including Compton scattering, but is convenient for calculating images that are relevant to the interpretation of EHT and GRAVITY data.

The results discussed below are based on simulations described in Fragile et al. (2007); Fragile (2009) and McKinney \& Blandford (2009); McKinney et al. (2012). Images are calculated over a large grid of parameters (viewing geometry, accretion rate, ion-electron temperature ratio), blurred to account for the effects of interstellar scattering towards Sgr A* (Bower et al. 2006), and fit to mm-VLBI (Doeleman et al. 2008; Fish et al. 2011) and spectral (Marrone et al. 2006) data.

\section{Summary of results}

Event horizon scale emission models of Sgr A* calculated from GRMHD simulations provide an excellent description of current sub-mm spectral and VLBI data (Dexter et al. 
2009, 2010). For a given model, it is possible to constrain the parameters of both the black hole and the accretion flow (Dexter et al. 2010), and this provides a new method for estimating e.g., the accretion rate onto Sgr A*. The best fitting images tend to have a "crescent" morphology (Bromley et al. 2001, see Figure 1), a combination of strong Doppler beaming and gravitational light bending. The images can even be crescentshaped for the complicated emission regions of accretion disks whose angular momentum is misaligned from the black hole spin axis (Dexter \& Fragile 2013). If the particles in the jet are close to the black hole, light from behind the black hole can be strongly lensed towards the observer (Dexter et al. 2012), forming a crescent image. Crescent images of disks and jets successfully predicted the results of mm-VLBI observations of M87 (Doeleman et al. 2012). These models predict that the black hole shadows in both Sgr A* and M87 may be accessible with additional radio telescopes joining EHT observations in the next few years. We have developed a geometric description of crescent black hole images (Bin Kamruddin \& Dexter 2013) and shown that it statistically outperforms other shapes (see also Broderick et al. 2011). We are now simulating future EHT observations using this model to determine when it can distinguish between physical models and detect the shadow and surrounding "photon ring" (Ricarte \& Dexter, in prep.), whose shape could be used to test the no-hair theorem (Johannsen \& Psaltis 2010).

The simulations are time-dependent, and so light curves can be calculated selfconsistently. Magnetic turbulence driven by the MRI qualitatively produces the observed sub-mm variability (Dexter et al. 2009, 2010). Misaligned accretion flows can form strong standing shocks (Fragile \& Blaes 2008; Generozov et al. 2013), heating electrons and producing highly variable ("flaring") near-infrared emission in agreement with observations (Dexter \& Fragile 2013). These models lead to centroid motions that should be detectable with GRAVITY, but which are more complicated than circular orbits.

\section{Discussion}

Event horizon scale emission models of Sgr A* calculated from GRMHD simulations of black hole accretion flows do an excellent job explaining mm-VLBI and spectral observations. However, significant uncertainties remain. Most work has assumed a thermal distribution of electrons with a fixed ion-electron temperature ratio. Alternative prescriptions can lead to much different images (Moscibrodzka \& Falcke 2013) and should be explored in the future. Numerical particle-in-cell MRI simulations (Riquelme et al. 2012) can in principle be used to calculate an equilibrium electron energy distribution.

The underlying dynamics are also uncertain. Most GRMHD simulations assume alignment between the accreting gas and black hole, and dynamically insignificant magnetic fields. It is unclear whether either of these is a good assumption for systems like Sgr A* and M87 (Dexter \& Fragile 2013; McKinney et al. 2012, 2013). Further, the properties of the large-scale accretion flow (e.g., the circularization radius and field strength), not captured in these simulations, are uncertain. Some aspects of the current models appear to be relatively robust to these uncertainties (e.g., crescent images) but others may not be (e.g., estimates of black hole spin).

The quality and quantity of EHT and GRAVITY data will be increasing rapidly in the next several years, but will still need to be interpreted in terms of models. Doing transformative science will require connecting accretion and jet physics and signatures of strong gravity to the non-imaging interferometric (closure phase and polarized visibilities, e.g., see Figure 1) and astrometric observables. These observables have been calculated from orbiting hotspot models (Hamaus et al. 2009; Doeleman et al. 2009; Fish et al. 2009), but should be explored using numerical simulations where the dynamics is 
treated self-consistently. It will also be important to leverage all existing observational constraints, e.g. on the polarization, time variability (Dexter et al. 2013), and X-ray luminosity (Neilsen et al. 2013; Wang et al. 2013). The calculations reviewed here provide a foundation for this future work.

\section{Acknowledgements}

I am grateful to several collaborators involved in this work, particularly E. Agol, P. C. Fragile, and J. C. McKinney. I also thank the participants of the stimulating discussion at this meeting about the Galactic center magnetar, which helped to inspire our recent work on the subject (Dexter \& O'Leary 2013).

\section{References}

Bardeen, J. M. 1973, in Black holes (Les astres occlus), ed. B. S. DeWitt \& C.DeWitt (New York: Gordon and Breach), 215

Bin Kamruddin, A., \& Dexter, J. 2013, MNRAS 434, 765

Bower, G. C., Goss, W. M., Falcke, H., Backer, D. C., \& Lithwick, Y. 2006, ApJ Lett. 648, L127

Broderick, A. E., Fish, V. L., Doeleman, S. S., \& Loeb, A. 2009, ApJ 697, 45

-. 2011, ApJ 735, 110

Bromley, B. C., Melia, F., \& Liu, S. 2001, ApJ Lett. 555, L83

De Villiers, J.-P., \& Hawley, J. F. 2003, ApJ 589, 458

Dexter, J. 2011, PhD thesis, University of Washington

Dexter, J., \& Agol, E. 2009, ApJ 696, 1616

Dexter, J., Agol, E., \& Fragile, P. C. 2009, ApJ Lett. 703, L142

Dexter, J., Agol, E., Fragile, P. C., \& McKinney, J. C. 2010, ApJ 717, 1092

Dexter, J., \& Fragile, P. C. 2013, MNRAS 432, 2252

Dexter, J., Kelly, B., Bower, G. C., et al. 2013, arXiv:1308.5968

Dexter, J., McKinney, J. C., \& Agol, E. 2012, MNRAS 421, 1517

Dexter, J., \& O'Leary, R. M. 2013, arXiv:1310.7022, 2014, ApJ 783L, 7

Dibi, S., Drappeau, S., Fragile, P. C., Markoff, S., \& Dexter, J. 2012, MNRAS 426, 1928

Doeleman, S. S., Fish, V. L., Broderick, A. E., Loeb, A., \& Rogers, A. E. E. 2009, ApJ 695, 59

Doeleman, S. S., Weintroub, J., Rogers, A. E.E., et al. 2008, Nature 455, 78

Doeleman, S. S., Fish, V. L., Schenck, D. E., et al. 2012, Science, 338, 355

Drappeau, S., Dibi, S., Dexter, J., Markoff, S., \& Fragile, P. C. 2013, MNRAS 431, 2872

Eisenhauer, F., Perrin, G., Brandner, W., et al. 2008, in Society of Photo-Optical Instrumentation Engineers (SPIE) Conference Series, Vol. 7013

Falcke, H., Melia, F., \& Agol, E. 2000, ApJ Lett. 528, L13

Fish, V. L., Doeleman, S. S., Broderick, A. E., Loeb, A., \& Rogers, A. E.E. 2009, ApJ 706, 1353

Fish, V. L., Doeleman, S. S., Beaudoin, C., et al. 2011, ApJ Lett. 727, L36

Fragile, P. C. 2009, ApJ Lett. 706, L246

Fragile, P. C., \& Blaes, O. M. 2008, ApJ 687, 757

Fragile, P. C., Blaes, O. M., Anninos, P., \& Salmonson, J. D. 2007, ApJ 668, 417

Gammie, C. F., McKinney, J. C., \& Tóth, G. 2003, ApJ 589, 444

Generozov, A., Blaes, O., Fragile, P. C., \& Henisey, K. B. 2013, ArXiv e-prints, 2014, ApJ 780, 81

Hamaus, N., Paumard, T., Müller, T., et al. 2009, ApJ 692, 902

Johannsen, T., \& Psaltis, D. 2010, ApJ 718, 446

Marrone, D. P., Moran, J. M., Zhao, J.-H., \& Rao, R. 2006, J. Phys. Conference Series, 54, 354

McKinney, J. C., \& Blandford, R. D. 2009, MNRAS 394, L126

McKinney, J. C., Tchekhovskoy, A., \& Blandford, R. D. 2012, MNRAS 423, 3083

-. 2013, Science, 339, 49

Moscibrodzka, M., \& Falcke, H. 2013, ArXiv e-prints, 2013, A\&A 4 559L, 3 
Mościbrodzka, M., Gammie, C. F., Dolence, J. C., Shiokawa, H., \& Leung, P. K. 2009, ApJ 706, 497

Neilsen, J., Nowak, M. A., Gammie, C., et al. 2013, ApJ 774, 42

Noble, S. C., Leung, P. K., Gammie, C. F., \& Book, L. G. 2007, Class. and Quant. Gravity, 24, 259

Riquelme, M. A., Quataert, E., Sharma, P., \& Spitkovsky, A. 2012, ApJ 755, 50

Shcherbakov, R. V., \& Huang, L. 2011, MNRAS 410, 1052

Shcherbakov, R. V., Penna, R. F., \& McKinney, J. C. 2012, ApJ 755, 133

Wang, Q. D., Nowak, M. A., Markoff, S. B., et al. 2013, Science, 341, 981 